\title{
Computational dynamics of a Lotka-Volterra Model with additive Allee effect based on Atangana-Baleanu fractional derivative
}

Hasan S. Panigoro and Emli Rahmi

To cite this article:

H. Panigoro and E. Rahmi,"Computational dynamics of a Lotka-Volterra Model with additive Allee effect based on Atangana-Baleanu fractional derivative", Jambura J. Biomath, vol. 2, no. 2, pp. 96-103, 2021

DOI: https://doi.org/10.34312/jjbm.v2i2.11886

(C) 2021 Author(s).

Articles You may be interested in

Fear effect in discrete prey-predator model incorporating square root functional response

P. K. Santra

http://dx.doi.org/10.34312/jjbm.v2i2.10444

Impact of predator fear on two competing prey species

D. Mukherjee

http://dx.doi.org/10.34312/jjbm.v2i1.9249

Dynamics in two competing predators-one prey system with two types of Holling and fear effect

A. L. Firdiansyah and N. Nurhidayati

http://dx.doi.org/10.34312/jjbm.v2i2.11264

Analisis dinamik model predator-prey tipe Gause dengan wabah penyakit pada prey

R. Ibrahim, L. Yahya, E. Rahmi, dan R. Resmawan

http://dx.doi.org/10.34312/jjbm.v2i1.10363

Dynamics of a stage-structure Rosenzweig-MacArthur model with linear harvesting in prey and cannibalism in predator

L. K. Beay and M. Saija

http://dx.doi.org/10.34312/jjbm.v2i1.10470 


\title{
Computational dynamics of a Lotka-Volterra Model with additive Allee effect based on Atangana-Baleanu fractional derivative
}

\author{
Hasan S. Panigoro ${ }^{1, *}$, Emli Rahmi ${ }^{2}$ \\ 1,2 Department of Mathematics, State University of Gorontalo, Bone Bolango 96119, Indonesia \\ ${ }^{*}$ Corresponding author: hspanigoro@ung.ac.id
}

\begin{abstract}
This paper studies an interaction between one prey and one predator following Lotka-Volterra model with additive Allee effect in predator. The Atangana-Baleanu fractional-order derivative is used for the operator. Since the theoretical ways to investigate the model using this operator are limited, the dynamical behaviors are identified numerically. By simulations, the influence of the order of the derivative on the dynamical behaviors is given. The numerical results show that the order of the derivative may impact the convergence rate, the occurrence of Hopf bifurcation, and the evolution of the diameter of the limit-cycle.
\end{abstract}

Keywords: Lotka-Volterra; Allee Effect; Atangana-Baleanu; Numerical Solution

\section{Introduction}

A relationship between preys with their predators has an important role to preserves the balance in an ecosystem. The presence of predators may endanger the existence of prey with their direct killing. To investigate this phenomenon, some scientists use mathematical tools to study several points of view such as dynamical behaviors and forecasting. At the present moment, a deterministic approach using differential equations becomes a well-known method for scientists. The classical one is given by A. J. Lotka [1] defined by

$$
\begin{aligned}
& \frac{d x}{d t}=a x-b x y, \\
& \frac{d y}{d t}=c x y-d y,
\end{aligned}
$$

where $x, y, a, b, c$, and $d$ represent the density of prey, the density of predator, the intrinsic growth rate of prey, the predation rate, the predator growth rate which converted from the predation process, and the predator death rate. For ecological purposes, model (1) is modified to cover the specific biological process in nature. For instance, see [2-7]. In this time, we study the dynamical behavior of a Lotka-Volterra model which assumes the growth rate of the predator decreases as the impact of the intraspecific competition and difficulty in finding mates. This condition was first introduced by Warder Cyle Allee in 1931 [8], and becomes popular nowadays, see [9-12]. By using the additive Allee effect [13-15] and applying it into predator growth, we obtain the following model.

$$
\begin{aligned}
& \frac{d x}{d t}=a x-b x y, \\
& \frac{d y}{d t}=c x y-d y-\frac{m y}{y+n},
\end{aligned}
$$

where $m$ and $n$ are the Allee constant which called strong Allee effect if $m>n$ and weak Allee effect when $0<m<n[13,16]$.

Since the present state for each population is naturally related which all of their previous condition, the operator using the fractional derivative becomes the best preference for most mathematicians. The famous fractional derivative which used in biological modeling are Riemann-Liouville [17], Caputo [18], Caputo-Fabrizio [19], and Atangana-Baleanu [20] operators. The kernel of Riemann-Liouville and Caputo derivatives are single and local which means that these two operators are considered does not suitable to describe the local dynamics [21]. 
Panigoro and Savitri [3] discuss the dynamics of model (2) using Caputo fractional derivative as the operator. In this paper, we study model (2) using a fractional derivative with Mittag-Leffler kernel known as AtanganaBaleanu operator. Atangana-Baleanu fractional derivative has a non-local and non-singular kernel to cover the limitation of Riemann-Liouville, Caputo, and Caputo-Fabrizio fractional derivative. The Atangana-Baleanu in Caputo sense $(\mathrm{ABC})$ fractional derivative is given by the following definition.

Definition 1. [20] Suppose $0<\alpha \leq 1$. The Atangana-Baleanu fractional integral and derivative in Caputo sense of order $-\alpha$ (ABC sense) are respectively defined by

$$
\begin{aligned}
{ }^{A B C} \mathcal{I}_{t}^{\alpha} f(t) & =\frac{1-\alpha}{\mathcal{N}(\alpha)} f(t)+\frac{\alpha}{\Gamma(\alpha) \mathcal{N}(\alpha)} \int_{0}^{t}(t-s)^{\alpha-1} f(s) d s, \\
A B C & \mathcal{D}_{t}^{\alpha} f(t)=\frac{\mathcal{N}(\alpha)}{1-\alpha} \int_{0}^{t} E_{\alpha}\left[-\frac{\alpha}{1-\alpha}(t-s)^{\alpha}\right] f^{\prime}(s) d s,
\end{aligned}
$$

where $t \geq 0, f \in C^{n}([0,+\infty), \mathbb{R}), E_{\alpha}$ is the Mittag-Leffler function defined by $E_{\alpha}(t)=\sum_{k=0}^{\infty} \frac{t^{k}}{\Gamma(\alpha k+1)}$, and $\mathcal{N}(\alpha)$ is a normalization function with $\mathcal{N}(0)=\mathcal{N}(1)=1$.

By following the similar procedure as in [21, 22], model (2) with ABC sense is

$$
\begin{aligned}
& { }^{A B C} \mathcal{D}_{t}^{\alpha} x=a x-b x y=F_{1}(x, y) \\
& { }^{A B C} \mathcal{D}_{t}^{\alpha} y=c x y-d y-\frac{m y}{y+n}=F_{2}(x, y)
\end{aligned}
$$

According to our literature review, model (3) has never been studied although this model is simple and classic. Therefore, we give the following results in the next sections. In Section 2, the existence and uniqueness condition of model (3) is given. In Section 3, we show numerically the dynamics of model (3). We finally give the conclusion at last Section of our article.

\section{Existence and Uniqueness}

To guarantee the validity of the model, the existence and uniqueness of model (3) is shown. As the preliminaries, the following theorem is given.

Theorem 2. [20] The following fractional differential equation

$$
A B C \mathcal{D}_{t}^{\alpha} f(t)=\Psi(t)
$$

can be written as

$$
f(t)=\frac{1-\alpha}{\mathcal{N}(\alpha)} \Psi(t)+\frac{\alpha}{\Gamma(\alpha) \mathcal{N}(\alpha)} \int_{0}^{t} \Psi(s)(t-s)^{\alpha-1} d s
$$

We start by investigating the kernels of model (3). We obtain the following theorem.

Theorem 3. The kernels $F_{i}(x, y), i=1,2$, are contracted and satisfy the Lipschitz conditions if $0 \leq \zeta_{1}<1$ and $0 \leq \zeta_{2}<1$ where $\|x\| \leq \sigma,\|y\| \leq \bar{\sigma}, \zeta_{1}=a+b \bar{\sigma}$, and $\zeta_{2}=c \sigma+d+\frac{m}{n}$,

proof. Let $x, \bar{x}, y$, and $\bar{y}$ are functions for $F_{i}(x, y), i=1,2$. Therefore, we obtain

$$
\begin{aligned}
\left\|F_{1}(x, y)-F_{1}(\bar{x}, \bar{y})\right\| & =\|(a x-b x y)-(a \bar{x}-b \bar{x} y)\| \\
& =\|a(x-\bar{x})-b y(x-\bar{x})\| \\
& \leq\|a(x-\bar{x})\|+\|b y(x-\bar{x})\| \\
& \leq a\|x-\bar{x}\|+b \bar{\sigma}\|x-\bar{x}\| \\
& =\zeta_{1}\|x-\bar{x}\|,
\end{aligned}
$$


and

$$
\begin{aligned}
\left\|F_{2}(x, y)-F_{2}(\bar{x}, \bar{y})\right\| & =\left\|\left(c x y-d y-\frac{m y}{y+n}\right)-\left(c x \bar{y}-d \bar{y}-\frac{m \bar{y}}{\bar{y}+n}\right)\right\| \\
& =\left\|c x(y-\bar{y})-d(y-\bar{y})-\left(\frac{m y}{y+n}-\frac{m \bar{y}}{\bar{y}+n}\right)\right\| \\
& =\left\|c x(y-\bar{y})-d(y-\bar{y})-m n\left(\frac{y-\bar{y}}{(y+n)(\bar{y}+n)}\right)\right\| \\
& \leq\|c x(y-\bar{y})\|+\|d(y-\bar{y})\|+\left\|m n\left(\frac{y-\bar{y}}{(y+n)(\bar{y}+n)}\right)\right\| \\
& \leq c \sigma\|y-\bar{y}\|+d\|y-\bar{y}\|+\frac{m}{n}\|y-\bar{y}\| \\
& =\zeta_{2}\|y-\bar{y}\|,
\end{aligned}
$$

where $\|\cdot\|$ is the Euclidean norm. From eqs. (6) and (7) the kernels $F_{i}(x, y), i=1,2$, satisfy the Lipschitz conditions. Moreover, since $0 \leq \zeta_{1}<1$ and $0 \leq \zeta_{2}<1$ then the kernels $F_{i}(x, y), i=1,2$, are also contracted.

Now, by employing fixed-point theorem, the existence of solution of model (3) is identified. Obeying Theorem 2, the following following Volterra-type integral equations are achieved.

$$
\begin{aligned}
& x(t)-x(0)=\frac{1-\alpha}{\mathcal{N}(\alpha)} F_{1}(x(t), y(t))+\frac{\alpha}{\mathcal{N}(\alpha) \Gamma(\alpha)} \int_{0}^{t}(t-s)^{\alpha-1} F_{1}(x(s), y(s)) d s, \\
& y(t)-y(0)=\frac{1-\alpha}{\mathcal{N}(\alpha)} F_{2}(x(t), y(t))+\frac{\alpha}{\mathcal{N}(\alpha) \Gamma(\alpha)} \int_{0}^{t}(t-s)^{\alpha-1} F_{2}(x(s), y(s)) d s,
\end{aligned}
$$

Equation (8) can be written by the following recursive formula

$$
\begin{aligned}
& x_{n}(t)=\frac{1-\alpha}{\mathcal{N}(\alpha)} F_{1}\left(x_{n-1}(t), y_{n-1}(t)\right)+\frac{\alpha}{\mathcal{N}(\alpha) \Gamma(\alpha)} \int_{0}^{t}(t-s)^{\alpha-1} F_{1}\left(x_{n-1}(s), y_{n-1}(s)\right) d s, \\
& y_{n}(t)=\frac{1-\alpha}{\mathcal{N}(\alpha)} F_{2}\left(x_{n-1}(t), y_{n-1}(t)\right)+\frac{\alpha}{\mathcal{N}(\alpha) \Gamma(\alpha)} \int_{0}^{t}(t-s)^{\alpha-1} F_{2}\left(x_{n-1}(s), y_{n-1}(s)\right) d s,
\end{aligned}
$$

with initial conditions $x_{0}(t)=x(0)$ and $y_{0}(t)=y(0)$. The successive terms which acquired by obeying eq. (9) given by

$$
\begin{aligned}
\Psi_{1, n}(t)= & x_{n}(t)-x_{n-1}(t) \\
= & \frac{1-\alpha}{\mathcal{N}(\alpha)}\left(F_{1}\left(x_{n-1}(t), y_{n-1}(t)\right)-F_{1}\left(x_{n-1}(t), y_{n-1}(t)\right)\right) \\
& +\frac{\alpha}{\mathcal{N}(\alpha) \Gamma(\alpha)} \int_{0}^{t}(t-s)^{\alpha-1}\left(F_{1}\left(x_{n-1}(s), y_{n-1}(s)\right)-F_{1}\left(x_{n-2}(s), y_{n-2}(s)\right)\right) d s, \\
\Psi_{2, n}(t)= & y_{n}(t)-y_{n-1}(t) \\
= & \frac{1-\alpha}{\mathcal{N}(\alpha)}\left(F_{2}\left(x_{n-1}(t), y_{n-1}(t)\right)-F_{2}\left(x_{n-2}(t), y_{n-2}(t)\right)\right) \\
& +\frac{\alpha}{\mathcal{N}(\alpha) \Gamma(\alpha)} \int_{0}^{t}(t-s)^{\alpha-1}\left(F_{2}\left(x_{n-1}(s), y_{n-1}(s)\right)-F_{2}\left(x_{n-2}(s), y_{n-2}(s)\right)\right) d s .
\end{aligned}
$$

Therefore, from eq. (10), we get

$$
x_{n}(t)=\sum_{i=1}^{n} \Psi_{1, i}(t), \text { and } y_{n}(t)=\sum_{i=1}^{n} \Psi_{2, i}(t) .
$$

The norm of eq. (10) together with eqs. (6) and (7) given by

$$
\begin{aligned}
\left\|\Psi_{1, n}(t)\right\| & \leq \frac{1-\alpha}{\mathcal{N}(\alpha)} F_{1}\left\|\Psi_{1, n-1}(t)\right\|+\frac{\alpha}{\mathcal{N}(\alpha) \Gamma(\alpha)} F_{1} \int_{0}^{t}\left\|\Psi_{1, n-1}(s)\right\|(t-s)^{\alpha-1} d s, \\
\left\|\Psi_{2, n}(t)\right\| & \leq \frac{1-\alpha}{\mathcal{N}(\alpha)} F_{2}\left\|\Psi_{2, n-1}(t)\right\|+\frac{\alpha}{\mathcal{N}(\alpha) \Gamma(\alpha)} F_{2} \int_{0}^{t}\left\|\Psi_{2, n-1}(s)\right\|(t-s)^{\alpha-1} d s .
\end{aligned}
$$

Finally, the following theorem arises. 
Theorem 4. Model (3) has a unique solution if we can find $t_{\max }$ such that

$$
\frac{(1-\alpha) \zeta_{i}}{\mathcal{N}(\alpha)}+\frac{t_{\max }^{\alpha} \zeta_{i}}{\mathcal{N}(\alpha) \Gamma(\alpha)}<1, i=1,2
$$

proof. Let $x(t)$ and $y(t)$ are assumed as bounded functions. According to Theorem 3, kernels $F_{1}(x, y)$ and $F_{2}(x, y)$ satisfy Lipschitz conditions and also contractions. Using eq. (12), the following inequalities are obtained.

$$
\begin{aligned}
& \left\|\Psi_{1, n}(t)\right\| \leq\left\|x_{0}\right\|\left(\frac{(1-\alpha) F_{1}}{\mathcal{N}(\alpha)}+\frac{t^{\alpha} F_{1}}{\mathcal{N}(\alpha) \Gamma(\alpha)}\right)^{n}, \\
& \left\|\Psi_{2, n}(t)\right\| \leq\left\|y_{0}\right\|\left(\frac{(1-\alpha) F_{2}}{\mathcal{N}(\alpha)}+\frac{t^{\alpha} F_{2}}{\mathcal{N}(\alpha) \Gamma(\alpha)}\right)^{n} .
\end{aligned}
$$

Therefore, the solution given by eq. (11) are exists and smooth since $\left\|\Psi_{1, n}(t)\right\| \rightarrow 0$ and $\left\|\Psi_{2, n}(t)\right\| \rightarrow 0$ as $n \rightarrow \infty$ and $t=t_{\max }$. To show that the functions which satisfy eq. (8) are the solutions of eq. (3), we suppose that

$$
\begin{aligned}
& x(t)-x(0)=x_{n}(t)-\Phi_{1, n}(t), \\
& y(t)-y(0)=y_{n}(t)-\Phi_{2, n}(t) .
\end{aligned}
$$

where $\Phi_{i, n}(t), i=1,2$ are the remainder terms of series solutions. The norm of $\Phi_{i, n}(t)$ satisfies

$$
\begin{aligned}
\left\|\Phi_{1, n}(t)\right\| \leq & \frac{1-\alpha}{\mathcal{N}(\alpha)}\left\|F_{1}(x(t), y(t))-F_{1}\left(x_{n-1}(t), y_{n-1}(t)\right)\right\| \\
& +\frac{\alpha}{\mathcal{N}(\alpha) \Gamma(\alpha)} \int_{0}^{t}\left\|F_{1}(x(s), y(s))-F_{1}\left(x_{n-1}(s), y_{n-1}(s)\right)\right\|(t-s)^{\alpha-1} d s, \\
\left\|\Phi_{2, n}(t)\right\| \leq & \frac{1-\alpha}{\mathcal{N}(\alpha)}\left\|F_{2}(x(t), y(t))-F_{2}\left(x_{n-1}(t), y_{n-1}(t)\right)\right\| \\
& +\frac{\alpha}{\mathcal{N}(\alpha) \Gamma(\alpha)} \int_{0}^{t}\left\|F_{2}(x(s), y(s))-F_{2}\left(x_{n-1}(s), y_{n-1}(s)\right)\right\|(t-s)^{\alpha-1} d s .
\end{aligned}
$$

By applying this relation iteratively, we get at $t=t_{\max }$

$$
\begin{aligned}
& \left\|\Phi_{1, n}(t)\right\| \leq\left(\frac{1-\alpha}{\mathcal{N}(\alpha)}+\frac{t_{\text {max }}^{\alpha}}{\mathcal{N}(\alpha) \Gamma(\alpha)}\right)^{n+1} \zeta_{1}^{n+1}, \\
& \left\|\Phi_{2, n}(t)\right\| \leq\left(\frac{1-\alpha}{\mathcal{N}(\alpha)}+\frac{t_{\text {max }}^{\alpha}}{\mathcal{N}(\alpha) \Gamma(\alpha)}\right)^{n+1} \zeta_{2}^{n+1} .
\end{aligned}
$$

Therefore, we obtain $\left\|\Phi_{i, n}(t)\right\| \rightarrow 0, i=1,2$ for $n \rightarrow \infty$. This means, when eq. (8) are satisfied by the functions, then they are the solutions of eq. (3). Furthermore, we will show that the solution is unique by suppossing that $x^{*}(t)$ and $y^{*}(t)$ are another solution of eq. (3). Thus, we obtain

$$
\begin{aligned}
x(t)-x^{*}(t)= & \frac{1-\alpha}{\mathcal{N}(\alpha)}\left(F_{1}(x(t), y(t))-F_{1}\left(x^{*}(t), y^{*}(t)\right)\right) \\
& +\frac{\alpha}{\mathcal{N}(\alpha) \Gamma(\alpha)} \int_{0}^{t}\left(F_{1}(x(n), y(n))-F_{1}\left(x^{*}(n), y^{*}(n)\right)\right)(t-s)^{\alpha-1} d s, \\
y(t)-y^{*}(t)= & \frac{1-\alpha}{\mathcal{N}(\alpha)}\left(F_{2}(x(t), y(t))-F_{2}\left(x^{*}(t), y^{*}(t)\right)\right) \\
& +\frac{\alpha}{\mathcal{N}(\alpha) \Gamma(\alpha)} \int_{0}^{t}\left(F_{2}(x(n), y(n))-F_{2}\left(x^{*}(n), y^{*}(n)\right)\right)(t-s)^{\alpha-1} d s .
\end{aligned}
$$

By utilizing the similar ways as in (12) and (14), eq. (18) become

$$
\begin{aligned}
& \left\|x(t)-x^{*}(t)\right\|\left(1-\frac{(1-\alpha) \zeta_{1}}{\mathcal{N}(\alpha)}-\frac{t^{\alpha} \zeta_{1}}{\mathcal{N}(\alpha) \Gamma(\alpha)}\right) \leq 0 \\
& \left\|y(t)-y^{*}(t)\right\|\left(1-\frac{(1-\alpha) \zeta_{2}}{\mathcal{N}(\alpha)}-\frac{t^{\alpha} \zeta_{2}}{\mathcal{N}(\alpha) \Gamma(\alpha)}\right) \leq 0
\end{aligned}
$$

For $t=t_{\max }$, we have eq. (13). Hence, $\left\|x(t)-x^{*}(t)\right\|=0$ and $\left\|y(t)-y^{*}(t)\right\|=0$ which conclude that $x(t)=$ $x^{*}(t)$ and $y(t)=y^{*}(t)$. Hence, model (3) has a unique solution. 


\section{Numerical Results}

In section, we perform some numerical simulations of model (3) including the bifurcation diagram, phase portraits, and time series. The predictor-corrector scheme developed by Baleanu et.al. [23] is utilized. The predictor for the solution of model (3) is given by the following equations.

$$
\begin{aligned}
& x_{i+1}^{P}=x_{0}+\frac{h_{N}^{\alpha}}{\mathcal{N}(\alpha) \Gamma(\alpha)} \sum_{j=0}^{i} \hat{b}_{i+1, j}^{(\alpha)} F_{1}\left(t_{j}, x_{j}, y_{j}\right), \\
& y_{i+1}^{P}=y_{0}+\frac{h_{N}^{\alpha}}{\mathcal{N}(\alpha) \Gamma(\alpha)} \sum_{j=0}^{i} \hat{b}_{i+1, j}^{(\alpha)} F_{2}\left(t_{j}, x_{j}, y_{j}\right),
\end{aligned}
$$

where

$$
\begin{aligned}
& b_{i+1, j}^{(\alpha)}=-(i-j)^{\alpha}+(i-j+1)^{\alpha}, \\
& \hat{b}_{i+1, j}^{(\alpha)}= \begin{cases}b_{i+1, j}^{(\alpha)} & , j=0, \ldots, i-1, \\
1+\frac{(1-\alpha) \Gamma(\alpha)}{h_{N}^{\alpha}}, j=i .\end{cases}
\end{aligned}
$$

while the corrector for the solution of model (3) is given by

$$
\begin{aligned}
& x_{i+1}=x_{0}+\frac{\alpha h_{N}^{\alpha}}{\mathcal{N}(\alpha) \Gamma(\alpha+2)}\left(\hat{a}_{i+1, i+1}^{(\alpha)} F_{1}\left(t_{i+1}, x_{i+1}^{P}, y_{i+1}^{P}\right)+\sum_{j=0}^{i} a_{i+1, j}^{(\alpha)} F_{1}\left(t_{i}, x_{j}, y_{j}\right)\right), \\
& y_{i+1}=y_{0}+\frac{\alpha h_{N}^{\alpha}}{\mathcal{N}(\alpha) \Gamma(\alpha+2)}\left(\hat{a}_{i+1, i+1}^{(\alpha)} F_{2}\left(t_{i+1}, x_{i+1}^{P}, y_{i+1}^{P}\right)+\sum_{j=0}^{i} a_{i+1, j}^{(\alpha)} F_{2}\left(t_{i}, x_{j}, y_{j}\right)\right),
\end{aligned}
$$

where

$$
\begin{aligned}
& \hat{a}_{i+1, i+1}^{(\alpha)}=1+\frac{(1-\alpha) \Gamma(\alpha+2)}{\alpha h_{N}^{\alpha}}, \\
& a_{i+1, j}^{(\alpha)}= \begin{cases}-(i-\alpha)(i+1)^{\alpha}+i^{\alpha+1} & , j=0 \\
(i-j)^{\alpha+1}-2(i-j+1)^{\alpha+1}+(i-j+2)^{\alpha+1} & , \quad 1 \leq j \leq i, \\
1 & , j=i+1 .\end{cases}
\end{aligned}
$$

The numerical error of this scheme is $\mathcal{O}^{q}$ where $q=\min (2,1+\alpha)$.

\subsection{Simulations}

Now, numerical simulations are performed. We choose the hypothetical parameter values: $a=0.5, b=0.3$, $c=0.25, d=0.1, m=0.1$, and $n=0.2$. By computing the equilibrium point, we have $(x, y) \approx(0.614,1.667)$ as the unique interior point. Since all parameters are fixed, we focus on observing the influence of the order of the derivative $\alpha$ to the dynamical behaviors of model (3) around the interior point. We first show the bifurcation diagram of model (3) in interval $0.92 \leq \alpha \leq 0.99$. The bifurcation diagram is given by Figure 1(a). For $0.92 \leq \alpha<0.943$, the interior point becomes stable. We show this condition by pick $\alpha=0.94$ and portray the phase portrait and time series in Figure $1(b, d)$. Furthermore, when $\alpha$ crosses 0.943 , the interior point becomes unstable and the solution tends to a periodic signal known as limit-cycle. This condition is maintained for $0.943<\alpha \leq 0.99$. We give an example for the solution by setting $\alpha=0.98$. The phase portrait and time series are performed in Figure 1(c,e). As in the model with Caputo sense shows in [3], this phenomenon is also happening which is called Hopf bifurcation where $\alpha$ is the bifurcation parameter and $\alpha=0.943$ is the bifurcation point. Therefore, Hopf bifurcation occurs both in Caputo and Atangana-Baleanu operators. In [21, 22], it has been shown numerically that the differences of Hopf bifurcation are driven by the order of the derivative given by Caputo and Atangana-Baleanu operator lie on the bifurcation point and the diameter of limit-cycle. To show the evolution of the diameter of the limit-cycle, we give Figure 2. The diameter of the limit-cycle increases when $\alpha \rightarrow 1$.

\subsection{Discussion}

From the numerical simulation, an interesting phenomenon has been shown. The changes in stability namely Hopf bifurcation occurs around the interior point. When the order of the derivative below of the bifurcation 


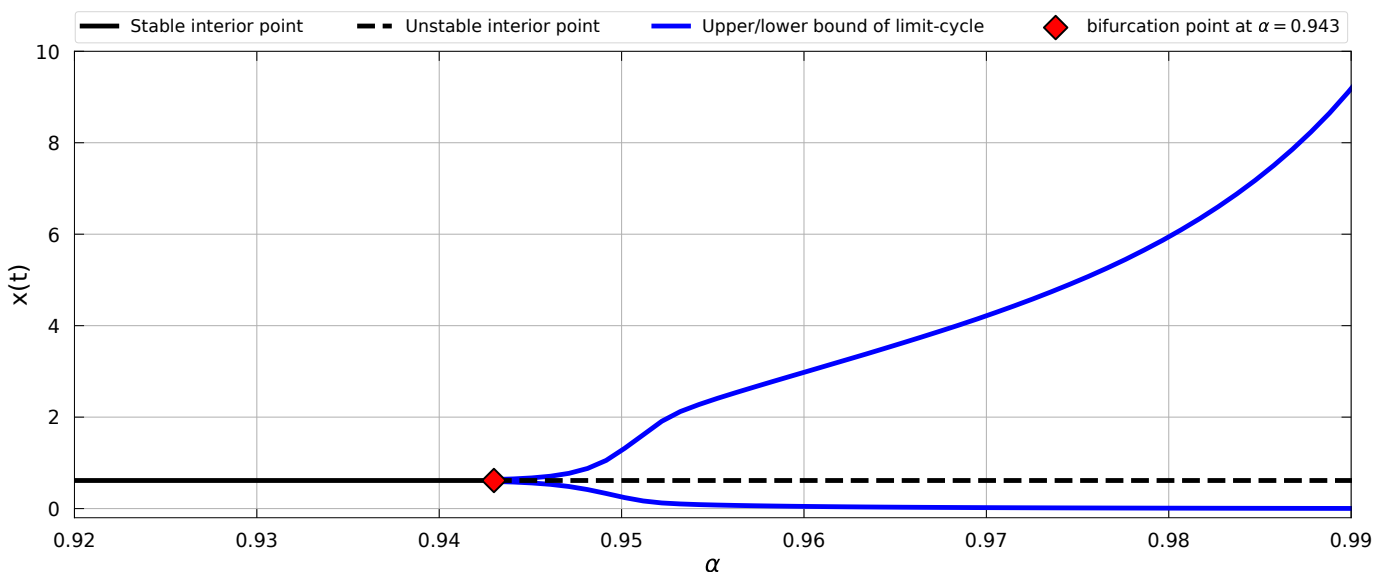

(a)

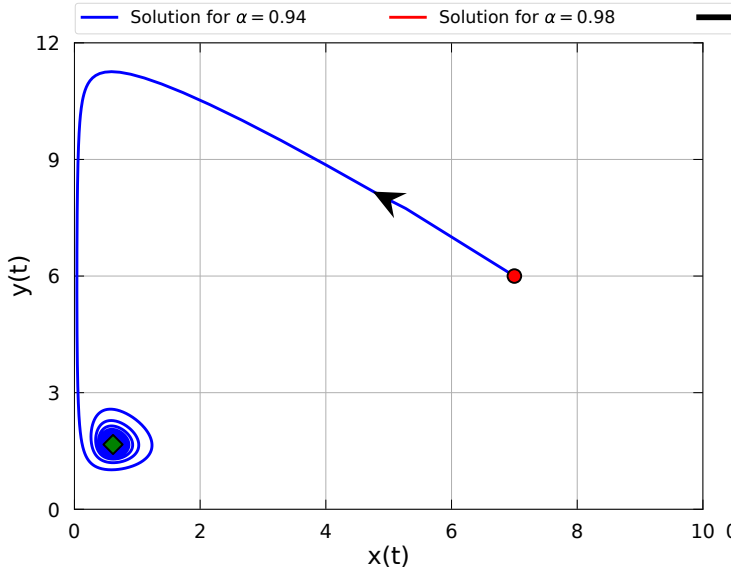

(b)

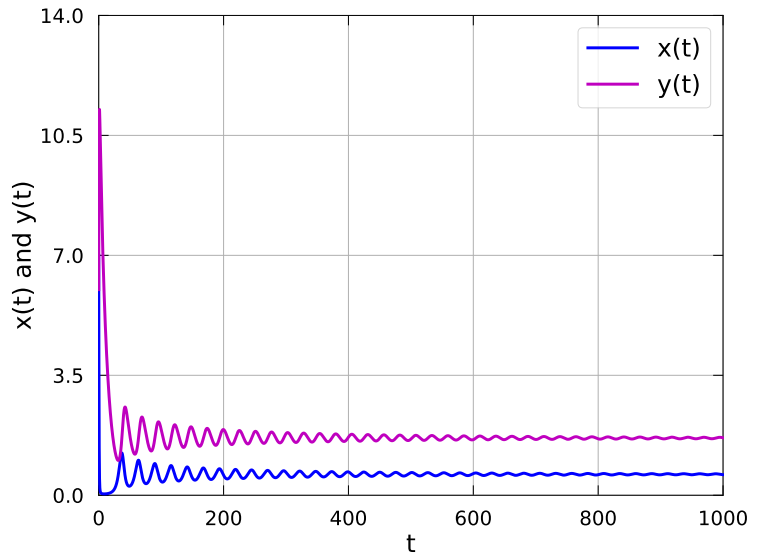

(d)

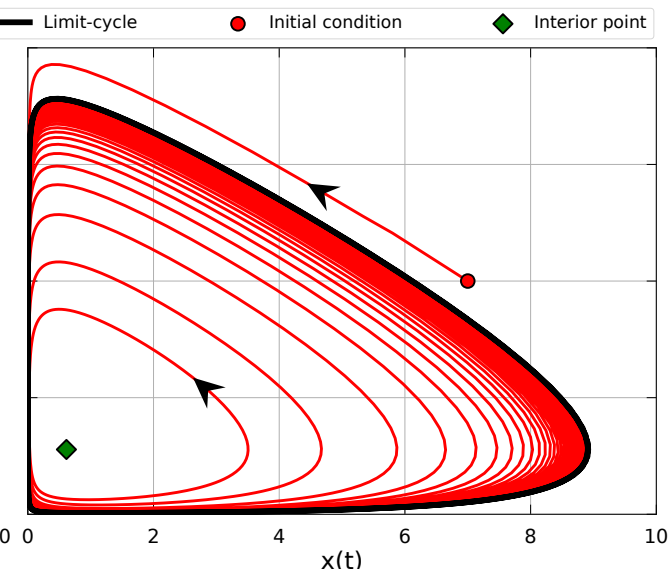

(c)

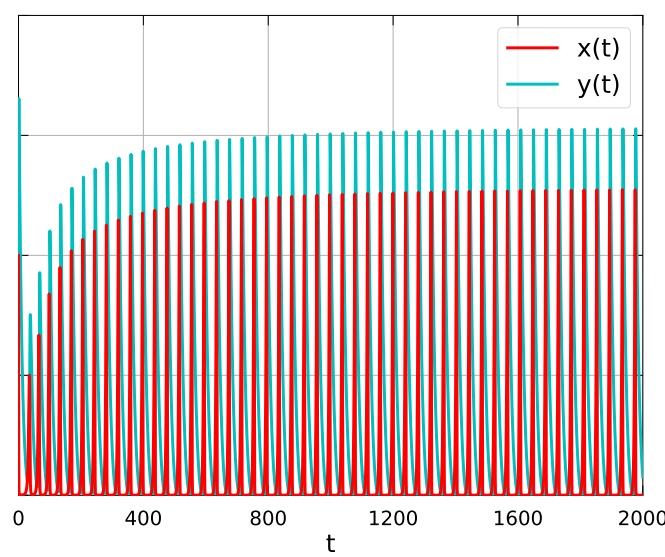

(e)

Figure 1. Numerical simulations of model (3) with parameter values: $a=0.5, b=0.3, c=0.25, d=0.1, m=0.1$, and $n=0.2$ (a) Bifurcation diagram driven by $\alpha$ in interval $[0.92,0.99]$ (b) Phase portrait when $\alpha=0.94$ (c) Phase portrait when $\alpha=0.98$ (d) Time series when $\alpha=0.94$ (e) Time series when $\alpha=0.98$

point, all nearby solutions converge to a unique point in the interior. This means the population densities of prey and predator eventually converge to positive constant values. Both populations managed to maintain their existence in nature constantly. When the order of the derivative above of the bifurcation point, the interior point losses its stability via bifurcation point which means all nearby solutions away from the interior point and converges to a periodic signal called limit-cycle. Although the density of prey and predator cannot be maintained constantly, both populations will never go extinct. The densities will eventually change periodically where the 


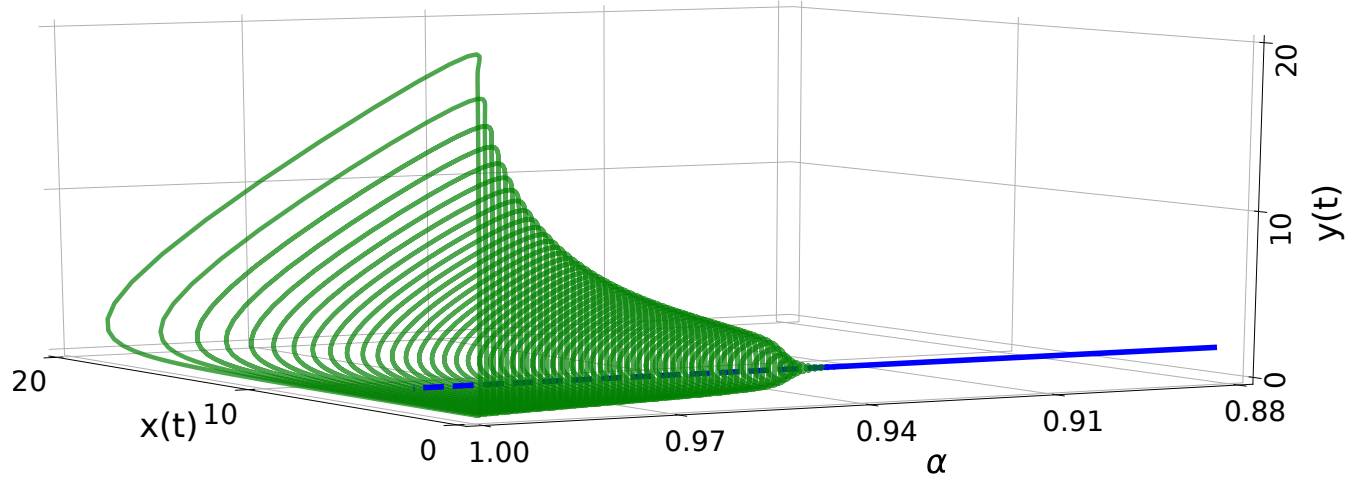

(a)

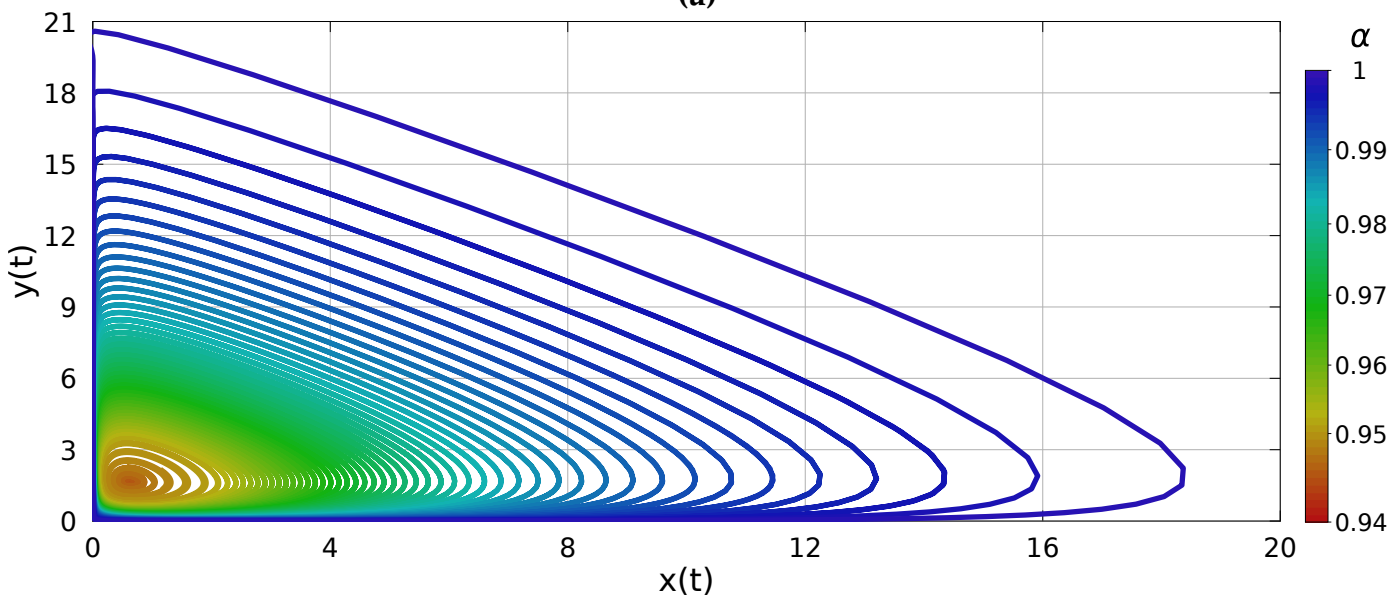

(b)

Figure 2. The evolution of limit-cycle (3) driven by $\alpha$ with parameter values : $a=0.5, b=0.3, c=0.25, d=0.1$, $m=0.1$, and $n=0.2$ (a) $3 D$-Bifurcation diagram in $(x, y, \alpha)$-space (b) The diameter evolution

evolution of their densities depends on the value of the order of the derivative. Since the order of the derivative means the index of memory, the behaviors of both prey and predator to maintain their existence depend on how all of their previous conditions affect them.

If we compare the numerical results given by Panigoro and Savitri [3] with model (3), although model with Caputo operator in [3] also undergoes Hopf bifurcation, in our works we show more detail about that bifucation phenomenon. The value of bifurcation point, the bifurcation diagram, and the evolution of limit-cycle have been presented numerically which does not exist in [3].

\section{Conclusion}

The dynamical behaviors of a Lotka-Volterra model with additive Allee effect involving Atangana-Baleanu fractional derivative have been studied numerically. The existence and uniqueness of the solution have been shown to demonstrate that the model is biologically viable. The predictor-corrector numerical scheme is successfully applied to the model to demonstrate the occurrence of Hopf bifurcation, the evolution of the diameter of the limit-cycle, and their suitable solutions. The numerical simulations also effectively demonstrate the impact of the memory on the prey and predator behaviors in maintaining their existence.

\section{References}

[1] A. J. Lotka, “Elements of Physical Biology," Nature, vol. 116, no. 2917, pp. 461-461, 1925.

[2] D. Savitri and H. S. Panigoro, "Bifurkasi Hopf pada model prey-predator-super predator dengan fungsi respon yang berbeda," Jambura Journal of Biomathematics, vol. 1, no. 2, pp. 65-70, dec 2020.

[3] H. S. Panigoro and D. Savitri, "Bifurkasi Hopf pada model Lotka-Volterra orde-fraksional dengan efek Allee aditif pada predator," Jambura Journal of Biomathematics, vol. 1, no. 1, pp. 16-24, 2020.

[4] L. K. Beay and M. Saija, "Dynamics of a stage-structure Rosenzweig-MacArthur model with linear harvesting in prey 
and cannibalism in predator," Jambura Journal of Biomathematics, vol. 2, no. 1, pp. 42-50, jun 2021.

[5] H. Deng, F. Chen, Z. Zhu, and Z. Li, "Dynamic behaviors of Lotka-Volterra predator-prey model incorporating predator cannibalism," Advances in Difference Equations, vol. 2019, no. 1, pp. 1-17, 2019.

[6] Z. Zhu, R. Wu, L. Lai, and X. Yu, "The influence of fear effect to the Lotka-Volterra predator-prey system with predator has other food resource," Advances in Difference Equations, vol. 2020, no. 1, pp. 1-13, 2020.

[7] S. Maisaroh, R. Resmawan, and E. Rahmi, "Analisis kestabilan model predator-prey dengan infeksi penyakit pada prey dan pemanenan proporsional pada predator," Jambura Journal of Biomathematics, vol. 1, no. 1, pp. 8-15, jun 2020.

[8] W. C. Allee, Animal aggregations, a study in general sociology. Chicago :: The University of Chicago Press,, 1931.

[9] C. Rebelo and C. Soresina, "Coexistence in seasonally varying predator-prey systems with Allee effect," Nonlinear Analysis: Real World Applications, vol. 55, p. 103140, 2020.

[10] E. Rahmi, I. Darti, A. Suryanto, Trisilowati, and H. S. Panigoro, "Stability Analysis of a Fractional-Order Leslie-Gower Model with Allee Effect in Predator," Journal of Physics: Conference Series, vol. 1821, no. 1, 2021.

[11] C. Arancibia-Ibarra and J. Flores, "Dynamics of a Leslie-Gower predator-prey model with Holling type II functional response, Allee effect and a generalist predator," Mathematics and Computers in Simulation, vol. 188, pp. 1-22, 2021.

[12] E. Rahmi, I. Darti, A. Suryanto, and Trisilowati, "A Modified Leslie-Gower Model Incorporating Beddington-DeAngelis Functional Response, Double Allee Effect and Memory Effect," Fractal and Fractional, vol. 5, no. 3, p. 84, 2021.

[13] H. S. Panigoro, E. Rahmi, N. Achmad, and S. L. Mahmud, "The Influence of Additive Allee Effect and Periodic Harvesting to the Dynamics of Leslie-Gower Predator-Prey Model," Jambura Journal of Mathematics, vol. 2, no. 2, pp. 87-96, 2020.

[14] L. Lai, Z. Zhu, and F. Chen, "Stability and Bifurcation in a Predator-Prey Model with the Additive Allee Effect and the Fear Effect," Mathematics, vol. 8, no. 8, p. 1280, 2020.

[15] Y. Cai, C. Zhao, W. Wang, and J. Wang, "Dynamics of a Leslie-Gower predator-prey model with additive Allee effect," Applied Mathematical Modelling, vol. 39, no. 7, pp. 2092-2106, 2015.

[16] D. Indrajaya, A. Suryanto, and A. R. Alghofari, "Dynamics of modified Leslie-Gower predator-prey model with Beddington-DeAngelis functional response and additive Allee effect," International Journal of Ecology and Development, vol. 31, no. 3, pp. 60-71, 2016.

[17] I. Podlubny, Fractional differential equations: an introduction to fractional derivatives, fractional differential equations, to methods of their solution and some of their applications. San Diego CA: Academic Press, 1999.

[18] M. Caputo, "Linear Models of Dissipation whose Q is almost Frequency Independent-II," Geophysical Journal International, vol. 13, no. 5, pp. 529-539, 1967.

[19] M. Caputo and M. Fabrizio, "A new definition of fractional derivative without singular kernel," Progress in Fractional Differentiation and Applications, vol. 1, no. 2, pp. 73-85, 2015.

[20] A. Atangana and D. Baleanu, "New fractional derivatives with nonlocal and non-singular kernel: Theory and application to heat transfer model," Thermal Science, vol. 20, no. 2, pp. 763-769, 2016.

[21] H. S. Panigoro, A. Suryanto, W. M. Kusumawinahyu, and I. Darti, "Dynamics of an Eco-Epidemic Predator-Prey Model Involving Fractional Derivatives with Power-Law and Mittag-Leffler Kernel," Symmetry, vol. 13, no. 5, p. 785, 2021.

[22] — " "A Rosenzweig-MacArthur model with continuous threshold harvesting in predator involving fractional derivatives with power law and mittag-leffler kernel," Axioms, vol. 9, no. 4, p. 122, 2020.

[23] D. Baleanu, A. Jajarmi, and M. Hajipour, "On the nonlinear dynamical systems within the generalized fractional derivatives with Mittag-Leffler kernel," Nonlinear Dynamics, vol. 94, no. 1, pp. 397-414, 2018.

(C)2021 by the Author(s). This article is an open access article distributed under the terms and conditions of the Creative Commons Attribution-NonComercial 4.0 International License. Editorial of JJBM: Department of Mathematics, State University of Gorontalo, Jln. Prof. Dr. Ing. B. J. Habibie, Bone Bolango 96119, Indonesia. 


\section{Submit your manuscript at http://ejurnal.ung.ac.id/}

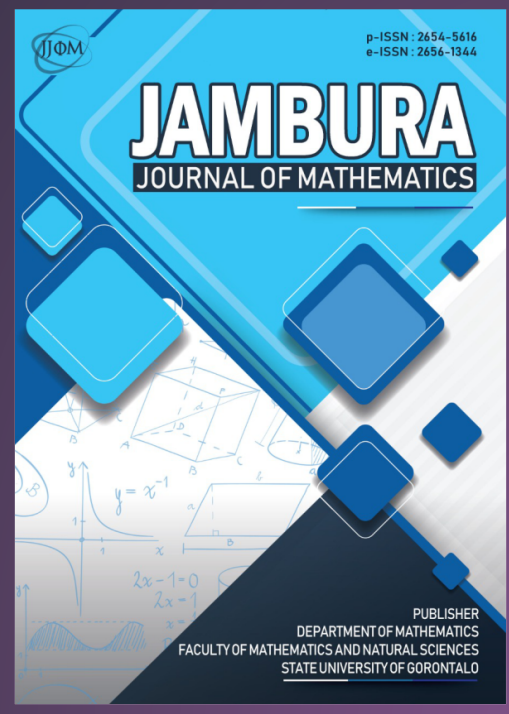

Jambura Journal of Mathematics

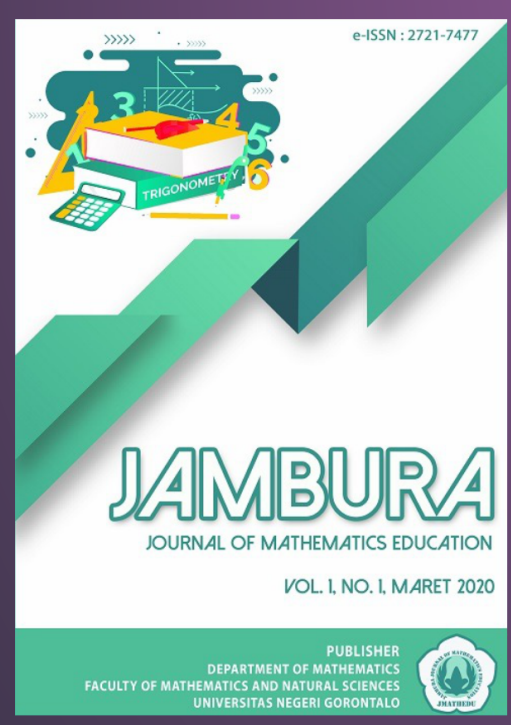

Jambura Journal of Mathematics Education
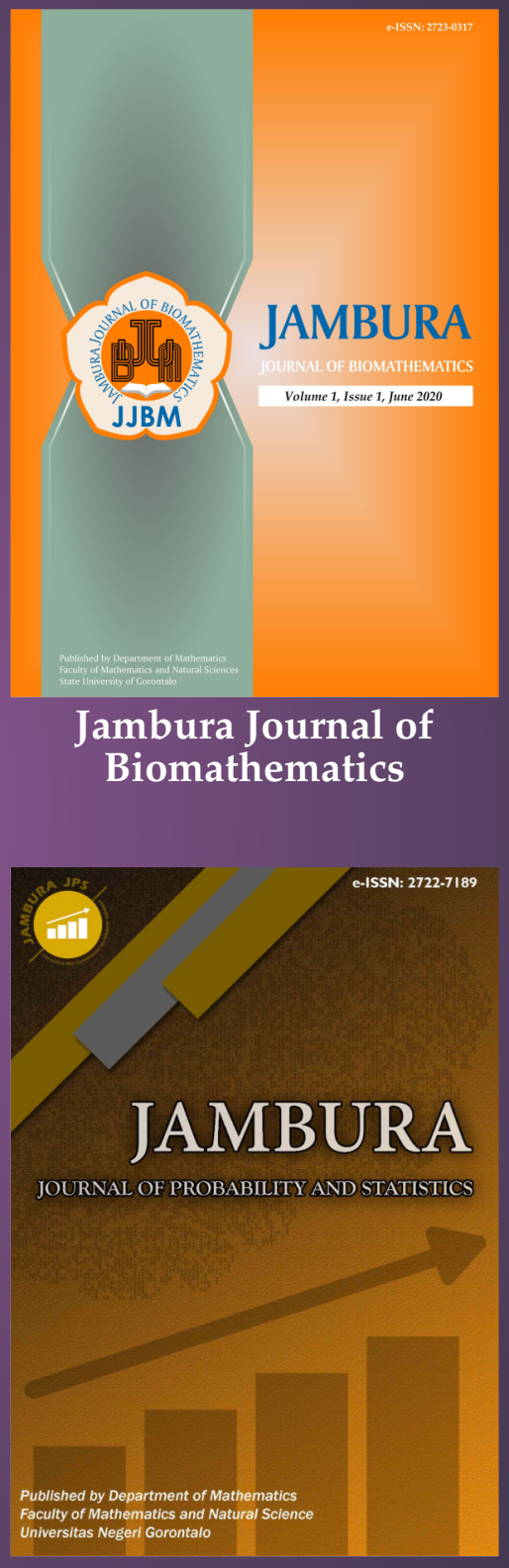

Jambura Journal of Probability and Statistics

Published by

Department of Mathematics

Faculty of Mathematics and Natural Sciences

State University of Gorontalo 